\title{
Correlations between structure, microstructure, density and dielectric properties of the lead-free ferroelectrics $\mathrm{Bi}_{0.5}(\mathrm{Na}, \mathrm{K})_{0.5} \mathrm{TiO}_{3}$
}

\author{
K. Anjali*,,,+ T. G. Ajithkumar ${ }^{\dagger, *}$ and P. A. Joy, $*, \S$ \\ *Physical and Materials Chemistry Division \\ ${ }^{\dagger}$ Central NMR Facility \\ *Academy of Scientific and Innovative Research (AcSIR) \\ CSIR-National Chemical Laboratory (CSIR-NCL) \\ Pune 411008, India \\ $\S$ pa.joy@ncl.res.in
}

Received 2 June 2015; Accepted 29 September 2015; Published 4 November 2015

\begin{abstract}
Solid solutions of the lead-free ferroelectric oxides $\mathrm{Bi}_{0.5} \mathrm{Na}_{0.5} \mathrm{TiO}_{3}$ (BNT) and $\mathrm{Bi}_{0.5} \mathrm{~K}_{0.5} \mathrm{TiO}_{3}(\mathrm{BKT})$, represented as $(1-x) \mathrm{BNT}-$ $x \mathrm{BKT}$, are studied for very close compositions in the range $0 \leq x \leq 0.36$ to understand the correlation between the structure and the properties. Compositions are varied in steps of $\Delta x=0.02$ in the range $0 \leq x \leq 0.1$ and $0.2 \leq x \leq 0.36$ and $\Delta x=0.01$ in the range $0.1 \leq x \leq 0.2$, to precisely locate the structural transition as well as the morphotropic phase boundary (MPB) region and the compositional region of best performance. Rietveld refinement analysis of the powder X-ray diffraction patterns showed monoclinic phase up to $x=0.17$ and a mixture of monoclinic and tetragonal phases for $x>0.17$. Similarly, the density and the dielectric constant showed linear variation up to $x=0.17$ and a large increase above this composition, showing maximum values in the compositional range $0.22 \leq x \leq 0.26$, corresponding to the MPB region. Microstructural features also showed corresponding changes, indicating close relationship between the structure, microstructure and properties of the different compositions.
\end{abstract}

Keywords: Bismuth sodium potassium titanate; $\mathrm{Bi}_{0.5} \mathrm{Na}_{0.5-x} \mathrm{~K}_{x} \mathrm{TiO}_{3}$; lead-free ferroelectrics; morphotropic phase boundary; structure-property correlations.

\section{Introduction}

The currently used piezoelectric ceramic compositions of lead zirconate titanate, $\mathrm{Pb}(\mathrm{Zr}, \mathrm{Ti}) \mathrm{O}_{3}(\mathrm{PZT})$, cause serious environmental pollution from the toxic metal, lead, present, ${ }^{1}$ even though they show high piezoelectric response and ferroelectric properties, ${ }^{2,3}$ for compositions near the morphotropic phase boundary (MPB). Therefore, it is necessary to develop alternate materials having satisfactory piezoelectric and ferroelectric properties. ${ }^{4}$ Lead-free bismuth-based titanates such as bismuth sodium titanate, $\mathrm{Bi}_{0.5} \mathrm{Na}_{0.5} \mathrm{TiO}_{3}$ (BNT) and bismuth potassium titanate, $\mathrm{Bi}_{0.5} \mathrm{~K}_{0.5} \mathrm{TiO}_{3}$ (BKT) are studied as promising ferroelectric ceramics with good piezoelectric characteristics, high electromechanical coupling factor and high Curie temperature, ${ }^{1,5-8}$ for various technological applications. Bismuth sodium titanate shows extended strain under high fields, ${ }^{9}$ which is almost comparable to that of PZT. This property makes BNT as an excellent candidate for the replacement of PZT in actuators even though the piezoelectric constant $\left(d_{33}\right)$ is considerably lower than that of PZT at low fields. Bismuth sodium titanate has large remnant polarization $\left(P_{r}=38 \mu \mathrm{C} / \mathrm{m}^{2}\right)$ and high Curie temperature $\left(T_{\mathrm{C}}=320^{\circ} \mathrm{C}\right){ }^{10,11}$ However, BNT has some drawbacks such as its high conductivity and large coercive field. This has been addressed by the substitution of $\mathrm{Na}$ by $\mathrm{K}$ in BNT.
In the solid solution series $\mathrm{Bi}_{0.5} \mathrm{Na}_{0.5-x} \mathrm{~K}_{x} \mathrm{TiO}_{3}$ (BNKT or BNT-BKT), or commonly represented as $(1-x)$ BNT$x \mathrm{BKT}$, it has been shown that the dielectric and piezoelectric properties are enhanced, with maximum electromechanical coupling constant in the region $x=0.16-0.20 .^{12,13}$

There are various contradicting reports about the crystal structure, structural phase transformation, existence of the MPB region and the compositional range of the MPB region of the $(1-x)$ BNT $-x$ BKT system. Even though the accepted space group of BNT at room temperature is rhombohedral $\mathrm{R} 3 \mathrm{c},{ }^{14}$ Gorfman and Thomas investigated the room temperature structure of BNT by using high resolution single crystal X-ray diffraction (XRD) and suggested that the actual symmetry is lower than rhombohedral, and achieved the best fit with the C-centered monoclinic lattice $(\mathrm{Cc}) .{ }^{15}$ Later, it has been reported that structure of polycrystalline BNT is best modeled using the monoclinic $\mathrm{Cc}$ space group rather than the previously accepted R3c space group. ${ }^{16}$ The room temperature crystal structure of BKT is tetragonal $(\mathrm{P} 4 \mathrm{~mm})$ and therefore, an MPB region is expected in the BNKT solid solution series, as observed for PZT, with different symmetries for the end members.

The presence of the MPB region is reported in the compositional range of $0.16 \leq x \leq 0.2$ in $(1-x) \mathrm{BNT}-x \mathrm{BKT}$ by

This is an Open Access article published by World Scientific Publishing Company. It is distributed under the terms of the Creative Commons Attribution 4.0 (CC-BY) License. Further distribution of this work is permitted, provided the original work is properly cited. 
Yang et al. from XRD analysis and electrical property measurements. ${ }^{17}$ Maximum density, relatively low grain size and compact microstructure with low porosity are obtained in this compositional region. Moreover, piezoelectric and dielectric properties are found to be maximum in this region. Kreisel et al. investigated the BNKT system using XRD and Raman spectroscopy, ${ }^{18}$ and it was observed that the crystal structure is rhombohedral for small $\mathrm{K}^{+}$concentrations $(x<0.25)$ and pseudo-cubic for $0.25 \leq x \leq 0.4$. Izumi et al. proposed that MPB region does not exist at all and only a structural distortion happens due to the local distortion in the A-site in the $\mathrm{ABO}_{3}$ perovskite crystal lattice. ${ }^{19}$ It was observed that the lattice parameters increase monotonically as a function of $\mathrm{K}^{+}$ concentration and the authors suggested that the compositions in the region $0.2 \leq x \leq 0.4$ are a mixture of rhombohedral and tetragonal domains, which leads to cubic-like XRD patterns. Sasaki et al. reported that the rhombohedral and tetragonal phases co-exist in the region $0.16 \leq x \leq 0.2$, based on the observation of a peak split in the XRD pattern. ${ }^{12}$ Chen et al. observed the presence of rhombohedral and tetragonal phases in the $x=0.18$ composition that was studied. ${ }^{20}$ Elkechai et al. found that rhombohedral and tetragonal phases co-exist in the compositional region $0.16 \leq x \leq 0.2$. $^{21}$ Xie et al. argued that the MPB region exists only at $x=0.2$ and the structure is rhombohedral and tetragonal below and above this value, respectively, and that there is no co-existence of a mixed phase in any compositional region. ${ }^{22}$

In all the reported studies on the structural aspects of the BNKT solid solution series, only few compositions have been studied in the MPB region. Therefore, to understand the structure-property correlations in this complicated system, a detailed structural study of the solid solution $(1-x)$ BNT$x$ BKT where $0 \leq x \leq 0.36$ with small compositional variations ( $\Delta x$ in steps of 0.02 for $0 \leq x \leq 0.1$ as well as $0.2 \leq$ $x \leq 0.36$ and in steps of 0.01 for $0.10 \leq x \leq 0.2$ ) have been carried out by Rietveld refinement of the powder XRD patterns and the changes in the structural parameters are correlated with the changes in the microstructure, density and dielectric constant. It is found that single phase compositions with monoclinic Cc structure exist for $x<0.18$. Large deviation in the measured properties is observed for $x>0.17$, which is correlated with the changes in the lattice parameters. Maximum density and dielectric constant are obtained for $0.22 \leq x \leq 0.26$ corresponding to a mixed phase composition containing monoclinic and tetragonal phases.

\section{Experimental Procedure}

Different compositions in the BNT-BKT solid solution series were prepared by the conventional solid-state reaction method using $\mathrm{Bi}_{2} \mathrm{O}_{3}(99.999 \%), \mathrm{Na}_{2} \mathrm{CO}_{3}(99.99 \%), \mathrm{K}_{2} \mathrm{CO}_{3}$ (99.99\%), $\mathrm{TiO}_{2}(99.999 \%)$. Stoichiometric amounts of the starting chemicals were taken according to the formula $(1-x) \mathrm{BNT}-x \mathrm{BKT}$, for $x$ ranging from 0 to 0.36 , and mixed thoroughly using an agate mortar and pestle. The mixed powders were initially calcined at $850^{\circ} \mathrm{C}$ and the pre-calcined powders were further calcined at $1000^{\circ} \mathrm{C}$ and $1150^{\circ} \mathrm{C}$, with an intermediate grinding. The powder samples were uniaxially pressed into circular discs $(10 \mathrm{~mm}$ diameter $\times 3 \mathrm{~mm}$ height) at a pressure of $8 \mathrm{MPa}$ and sintered at $1150^{\circ} \mathrm{C}$ for $2 \mathrm{~h}$. All the samples were sintered under identical conditions with heating and cooling rates of $5^{\circ} \mathrm{C} / \mathrm{min}$.

Structural characterizations of the sintered samples were performed by powder XRD on a PANalytical X'Pert PRO powder X-ray diffractometer. The densities of the sintered pellets were measured by the Archimedes method. Dielectric constants of the sintered pellets were measured at room temperature, using an impedance bridge (Model $1608 \mathrm{~A}$, General Radio Company) at $1 \mathrm{kHz}$. The microstructures of the sintered samples were obtained using a scanning electron microscope (FEI Quanta 200 3D ESEM). Rietveld refinement of the powder XRD patterns was carried using the software General Structure Analysis System (GSAS-EXPGUI). ${ }^{23}$

\section{Results and discussion}

Figure 1 shows the powder XRD patterns of selected compositions in the $(1-x) \mathrm{BNT}-x \mathrm{BKT}$ series. The simulated XRD pattern of BNT (using the rhombohedral R3c space group and the crystallographic parameters taken from Ref. 14) is also shown in the figure for comparison. All the XRD patterns show peaks corresponding to the perovskite lattice without any impurity peaks. Figure 2 compares the result of the Rietveld refinement analysis of BNT using the rhombohedral, R3c and monoclinic, $\mathrm{Cc}$, space groups. The R3c space group gave a good fit with $\chi^{2}=2.26$ and $\mathrm{R}_{p}=5.91 \%$. However, the monoclinic space group gave a better fit with improved $\chi^{2}=1.95$ and $\mathrm{R}_{p}=5.60 \%$. These

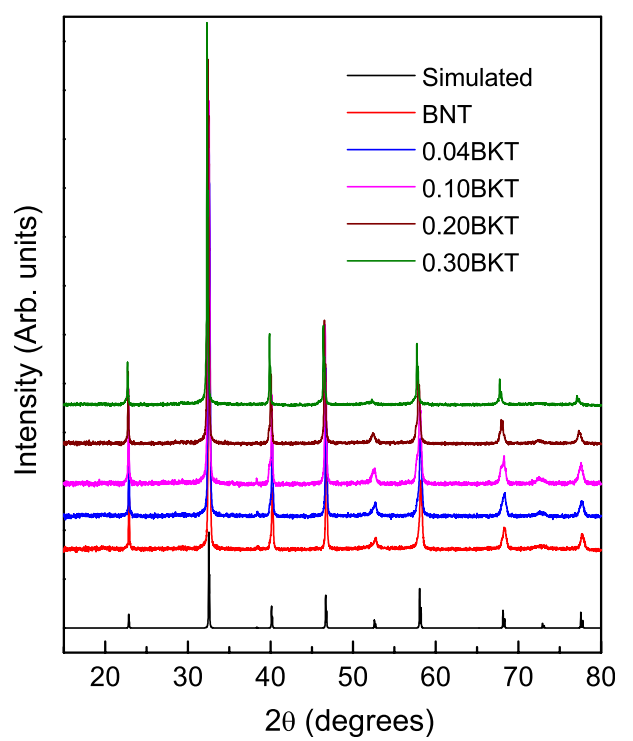

Fig. 1. XRD patterns of selected compositions in the $(1-x)$ BNT$x$ BKT system. 

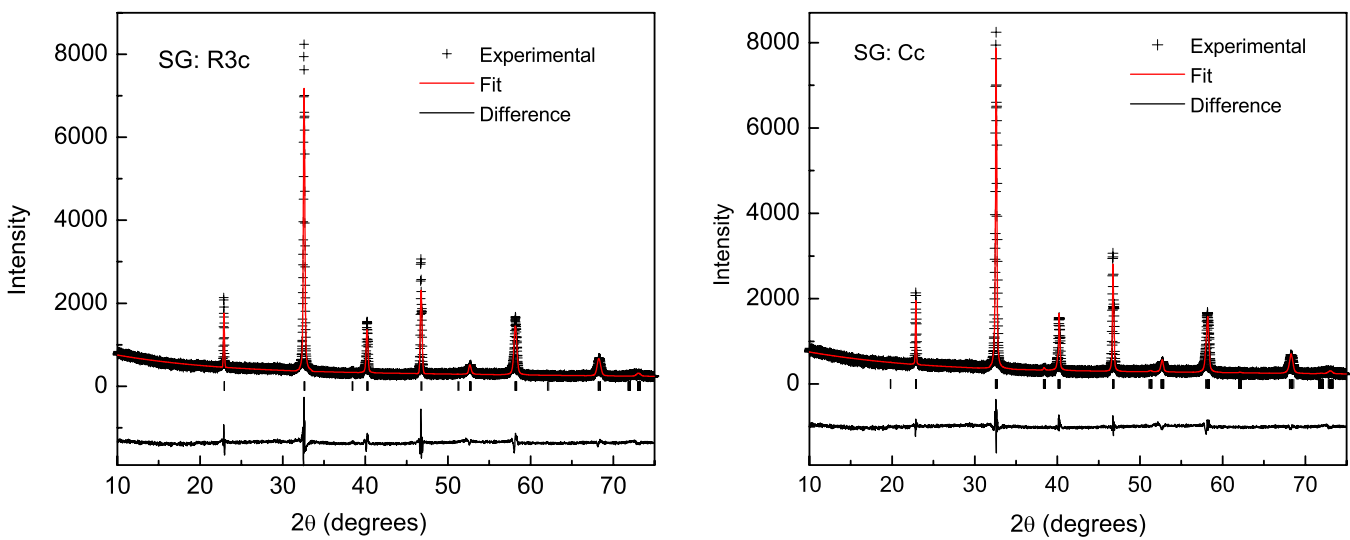

Fig. 2. Results of the Rietveld refinement analysis for BNT using rhombohedral R3c and monoclinic Cc space groups.

results suggest that the correct structure of BNT is monoclinic with space group Cc, as reported by Aksel et al. ${ }^{16}$

Structural refinement of different compositions for $x>0$ was carried out using the $\mathrm{Cc}$ space group. It was found that $\chi^{2}$ increases drastically for compositions $x>0.17$. The results indicate that on increasing the BKT concentration above $x=0.17$, the crystal symmetry is probably changing from monoclinic (Cc space group) to some other symmetry to accommodate the larger $\mathrm{K}^{+}$ion in the crystal lattice of BNT. Since the ionic radius of $\mathrm{K}^{+}(1.64 \AA)$ is much larger than that of $\mathrm{Na}^{+}(1.39 \AA)$, substitution of $\mathrm{Na}^{+}$by $\mathrm{K}^{+}$expands the lattice spacing of BNT, and hence the unit cell dimensions are expected to increase. The variation of the lattice parameters with increasing BKT content indicated interesting features. Most prominent change is observed for the lattice parameter ' $b$ ', as shown in Fig. 3. A linear increase in the value of ' $b$ ' is observed initially which starts deviating for $x>0.17$. These

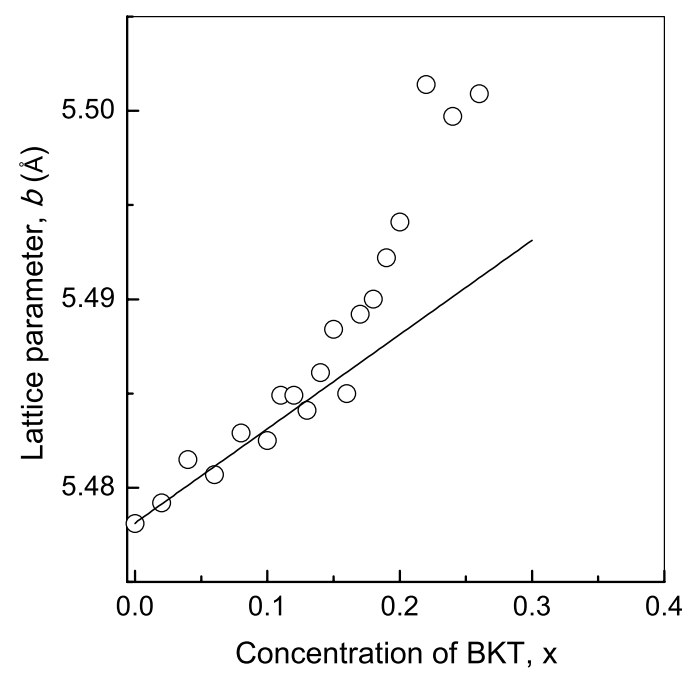

Fig. 3. Variation of the monoclinic lattice parameter ' $b$ ', as a function of $x$ in the $(1-x)$ BNT $-x$ BKT system. The solid line is straight line fit to the data for $0 \leq x \leq 0.17$. results suggest that the compositions in the range $0 \leq x \leq$ 0.17 form under the Cc space group and the structure and/or space group may be different for compositions $x>0.17$.

Since the XRD patterns of the higher BKT concentrations $(0.18 \leq x \leq 0.36)$ did not give good fit when the monoclinic Cc space group was used, Rietveld refinements for higher BKT concentrations $(0.18 \leq x \leq 0.36)$ were tried using the tetragonal space groups $\mathrm{P} 4 \mathrm{bm}$ as well as $\mathrm{P} 4 \mathrm{~mm}$ reported for BKT and the rhombohedral space group R3c. However, all of the compositions gave larger $\mathrm{R}_{p}$ and $\chi^{2}$ values, indicating that the tried structures are not the right models. Therefore, further refinements were tried with mixed phases $(\mathrm{R} 3 \mathrm{c}$ as well as $\mathrm{Cc}$ with $\mathrm{P} 4 \mathrm{bm}$ and $\mathrm{P} 4 \mathrm{~mm}$ space groups) which gave much improved fits, with relatively lower values of $\chi^{2}$ and $R_{p}$ for the mixed phases of $\mathrm{Cc}$ and $\mathrm{P} 4 \mathrm{bm}$. Figure 4 shows the results of the Rietveld refinement for $x=0.24$ using the monoclinic $\mathrm{Cc}$ space group alone and mixed phase of monoclinic $\mathrm{Cc}$ and tetragonal P4bm space groups. Thus, the XRD analysis results show that for the compositional region studied in the BNT-BKT solid solution series $(0 \leq x \leq 0.36)$, the structures are different for lower and higher values of $x$. Single phase compositions (monoclinic Cc space group) are formed for $x<0.18$ and the compositions in the range $0.18 \leq x \leq 0.36$, are found to be a mixture of monoclinic and tetragonal phases. From Rietveld refinement analysis, the composition with $x=0.18$ is found to be a mixture of $77 \%$ of the monoclinic phase and $23 \%$ of the tetragonal phase. For $x=0.36$, the phase content is obtained as $32 \%$ of the monoclinic phase and $68 \%$ of the tetragonal phase. Thus, the content of the tetragonal phase varied between $23 \%$ and $68 \%$, with a corresponding decrease in the monoclinic phase content, with increasing $x$, for $0.18 \leq x \leq 0.36$. There is no compositional region separating the two different structures, contrary to the previous report of observing a tetragonal structure alone above $x=0.18$.

SEM images of different compositions are compared in Fig. 5. Very large grains of size $\sim 10 \mu \mathrm{m}$ are observed for BNT $(x=0)$ with large inter- and intra-granular pores. The 


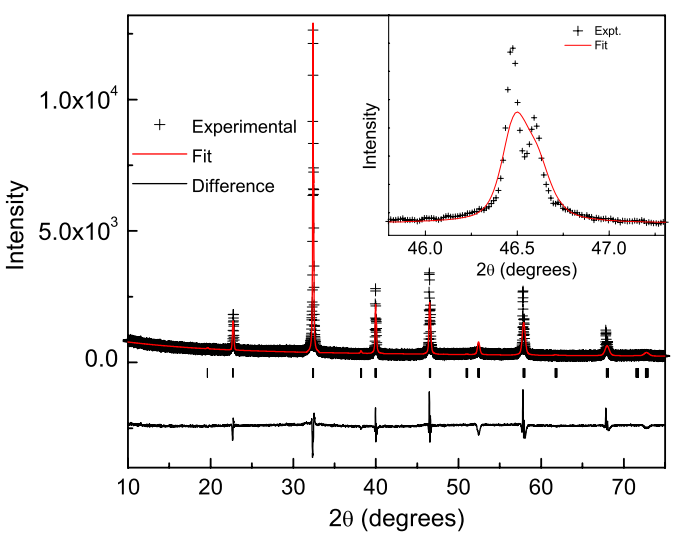

(a)

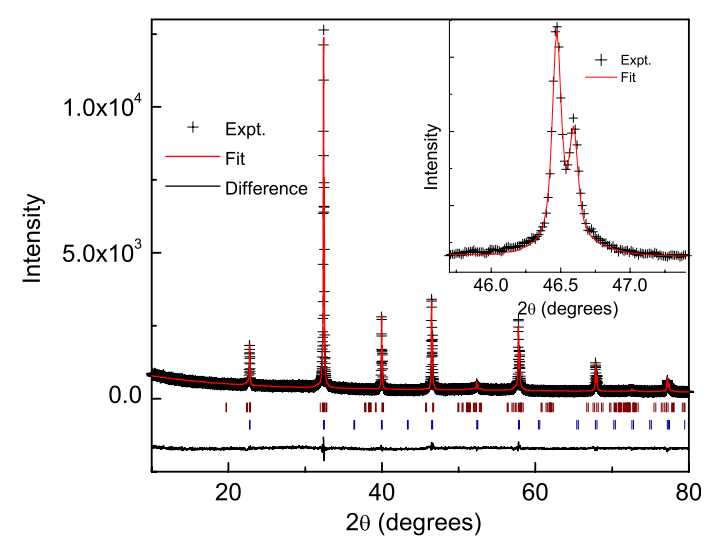

(b)

Fig. 4. Results of the Rietveld refinement analysis for $x=0.24$ using (a) monoclinic phase and (b) mixed monoclinic and tetragonal phases.

grain size as well as porosity are found to decrease with increasing BKT concentration. Individual grains of size in the range $1-5 \mu \mathrm{m}$ are clearly seen for the composition $x=0.16$. Further increase in the BKT concentration leads to the formation of fused grains, with decreasing porosity, for compositions in the range $0.18 \leq x \leq 0.24$. For compositions with $x>0.24$, individual and well-crystallized small grains are observed. Thus, there is a correlation between the phase content in the BNT-BKT system obtained from XRD analysis and the microstructure of the sintered products. Density and dielectric constant measurements have been carried out for all samples to study the correlation between the measured properties and the changes in the crystal structure as well as the microstructural features.

Figure 6 shows the variation of the sintered density of $(1-x) \mathrm{BNT}-x \mathrm{BKT}$ with BKT concentration. The density is relatively low, compared to the previous reports, and this is due to the lower pressure (8 MPa) used in the present work for compaction of the pellets. The density initially increases linearly with increasing $x$, suggesting the formation of an ideal solid solution. A large increase in the density is observed above $x=0.18$ and the density reaches a maximum value in the compositional region with $0.22 \leq x \leq 0.24$. The density is decreased above $x=0.24$ and reaches almost constant value for $x>0.26$. The changes in the density correlate very well with the features observed in the microstructure, where grain size and porosity decreases with increasing $x$ up to $x=0.18$, fused grains with increasing grain size are observed for compositions in the range $0.22 \leq x \leq 0.24$ and well-crystallized grains are observed for $x \geq 0.24$.

Figure 7 shows the variation of the dielectric constant of $(1-x)$ BNT $-x$ BKT as a function of $x$. It is observed that, on

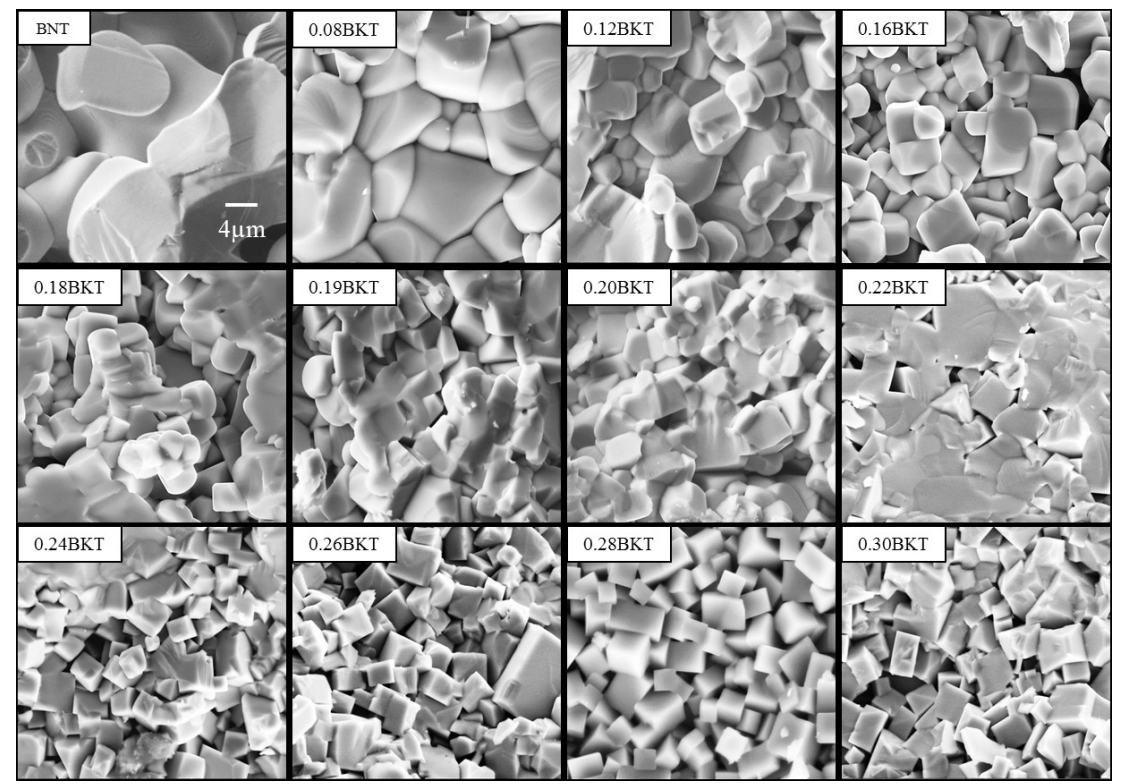

Fig. 5. SEM images of different compositions in the $(1-x)$ BNT $-x$ BKT system. The scale shown is common for all images. 


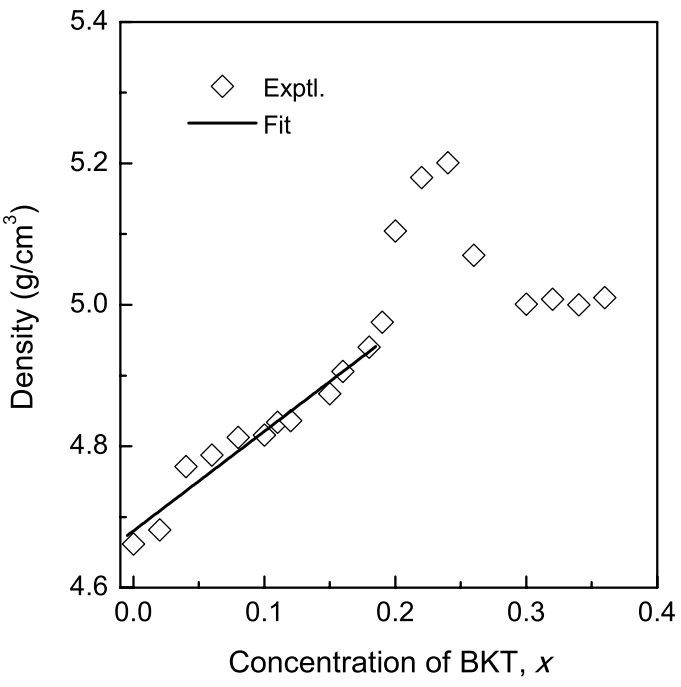

Fig. 6. Variation of the density of $(1-x) \mathrm{BNT}-x \mathrm{BKT}$ as a function of $x$. The solid line is straight line fit to the data for $0 \leq x \leq 0.17$.

increasing the BKT concentration, the dielectric constant of the material initially increases almost linearly up to $x=0.17$. A large increase in the dielectric constant is observed above $x>0.17$ and the value reaches a maximum in the compositional range $0.20 \leq x \leq 0.24$, with the highest value obtained for $x=0.24$. A sharp drop in the dielectric constant is observed above $x=0.24$ and the dielectric constant decreases almost linearly above $x=0.26$. Otonicar $e t$ al. have reported a maximum dielectric constant for $x=0.2{ }^{24}$ Thus, the present results show that the changes in the structure, phase content, microstructure, sintered density and dielectric constant of the $(1-x)$ BNT $-x$ BKT system follow exactly similar trend. A linear variation of the lattice parameter, density and dielectric constant are observed for $0 \leq x \leq 0.17$ and maximum values of density and dielectric constant are obtained in

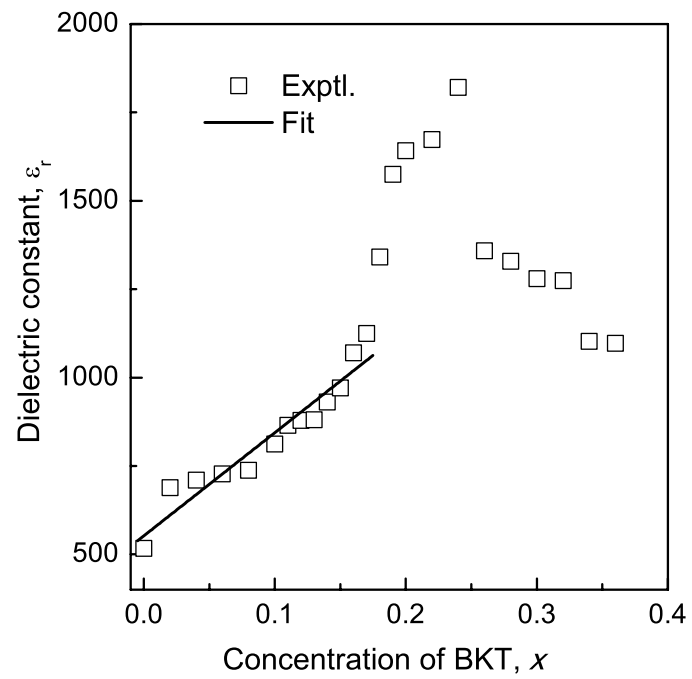

Fig. 7. Variation of the dielectric constant of $(1-x) \mathrm{BNT}-x \mathrm{BKT}$ as a function of $x$. The solid line is straight line fit to the data for $0 \leq x \leq 0.17$. the range $0.20 \leq x \leq 0.24$, where the microstructure shows fused grains. These results indicate the direct correlation between the changes in the structural, microstructural and performance parameters with increasing BKT content in the $(1-x)$ BNT $-x$ BKT system.

\section{Conclusions}

Structural, microstructural, density and dielectric properties of the lead-free ferroelectric compositions in the solid solution series $(1-x) \mathrm{Bi}_{0.5} \mathrm{Na}_{0.5} \mathrm{TiO}_{3}-(x) \mathrm{Bi}_{0.5} \mathrm{~K}_{0.5} \mathrm{TiO}_{3}$, represented as $(1-x)$ BNT $-x$ BKT, are studied to understand the correlation between the structure and properties of the system. From Rietveld refinement analysis of the powder XRD patterns, it is found that the crystal structure is monoclinic for $x<0.18$. A biphasic MPB region exists for $x \geq 0.18$ with monoclinic and tetragonal structures. There is a direct correlation between the structure, microstructure and density with the dielectric constant, where the properties vary linearly for $x<0.18$, deviates above $x=0.18$ and reached a maximum in the compositional region $0.22 \leq x \leq 0.28$ where the material shows maximum density. From the studies, it is concluded that better performance parameters can be obtained for $(1-x)$ BNT $-x$ BKT in the MPB region.

\section{Acknowledgments}

K. Anjali is grateful to University Grants Commission (UGC), India, for a research fellowship.

\section{References}

${ }^{1}$ I. Coondoo, N. Panwar and A. Kholkin, Lead-free piezoelectrics: Current status and perspectives, J. Adv. Dielect. 3, 1330002 (2013).

${ }^{2}$ A. V. ShilNikov, I. V. Otsarev, A. I. Burkhanov, V. N. Nesterov and G. M. Akbaeva, Dielectric properties of PZT-based compositions on the morphotropic phase boundary, Ferroelectrics 247, 205 (2000).

${ }^{3}$ T. Yamamoto and H. Moriwake, Ferroelectric properties and crystal structure of the $\mathrm{Pb}\left(\mathrm{Zr}_{1-x} \mathrm{Ti}_{x}\right) \mathrm{O}_{3}, x=0.200$ to 0.750 ceramics, J. Korean Phys. Soc. 32, S1301 (1998).

${ }^{4} \mathrm{~W}$. Liu and X. Ren, Large piezoelectric effect in Pb-free ceramics, Phys. Rev. Lett. 103, 257602 (2009).

${ }^{5}$ G. A. Smolenskii, V. A. Isupov, A. I. Agranovskaya and N. N. Krainik, New ferroelectrics of complex composition, Sov. Phys. Solid State 2, 2584 (1961).

${ }^{6}$ G. A. Smolenskii, Physical phenomena in ferroelectrics with diffused phase transitions, Jpn. J. Phys. Soc. 28, 26 (1970).

${ }^{7}$ T. Takenaka and K. Sakata, Dielectric, piezoelectric and pyroelectric properties of $(\mathrm{BiNa})_{1 / 2} \mathrm{TiO}_{3}$-based ceramics, Ferroelectrics 95, 153 (1989).

${ }^{8}$ T. Takenaka, K. Maruyama and K. Sakata, $\left(\mathrm{Bi}_{1 / 2} \mathrm{Na}_{1 / 2}\right) \mathrm{TiO}_{3^{-}}$ $\mathrm{BaTiO}_{3}$ system for lead-free piezoelectric ceramics, Jpn. J. Appl. Phys. 30, 2236 (1991). 
${ }^{9}$ D. Schutz, M. Deluca, W. Krauss, A. Feteira, T. Jackson and K. Reichmann, Lone-pair-induced covalency as the cause of temperature and field-induced instabilities in bismuth sodium titanate, Adv. Funct. Mater. 22, 2285 (2012).

${ }^{10}$ Y. Hiruma, H. Nagata and T. Takenaka, Phase diagram and electrical properties of $\mathrm{Bi}_{1 / 2} \mathrm{Na}_{1 / 2} \mathrm{TiO}_{3}$-based solid solutions, J. Appl. Phys. 104, 124106 (2008).

${ }^{11}$ A. J. Royles, A. J. Bell, J. E. Daniels, S. J. Milne and T. P. Comyn, Observation of a time-dependent structural phase transition in potassium sodium bismuth titanate, Appl. Phys. Lett. 98, 182904 (2011).

${ }^{12}$ A. Sasaki, T. Chiba, Y. Mamiya and E. Otsuki, Dielectric and piezoelectric properties of $\mathrm{Bi}_{0.5} \mathrm{Na}_{0.5} \mathrm{TiO}_{3}-\mathrm{Bi}_{0.5} \mathrm{~K}_{0.5} \mathrm{TiO}_{3}$ systems, Jpn. J. Appl. Phys. 38, 5564 (1999).

${ }^{13}$ A. Moosavi, M. A. Bahrevar, A. R. Aghaei, P. Ramos, M. Alguero and $\mathrm{H}$. Amorin, High-field electromechanical response of $\mathrm{Bi}_{0.5} \mathrm{Na}_{0.5} \mathrm{TiO}_{3}-\mathrm{Bi}_{0.5} \mathrm{~K}_{0.5} \mathrm{TiO}_{3}$ across its morphotropic phase boundary, J. Phys. D: Appl. Phys. 47, 055304 (2014).

${ }^{14} \mathrm{G}$. O. Jones and P. A. Thomas, Investigation of the structure and phase transitions in the novel A-site substituted distorted perovskite compound $\mathrm{Na}_{0.5} \mathrm{Bi}_{0.5} \mathrm{TiO}_{3}$, Acta. Crystallogr. B 58, 168 (2002).

${ }^{15} \mathrm{~S}$. Gorfman and P. A. Thomas, Evidence for a non-rhombohedral average structure in the lead-free piezoelectric material $\mathrm{Na}_{0.5} \mathrm{Bi}_{0.5} \mathrm{TiO}_{3}$, J. Appl. Crystallogr. 43, 1409 (2010).

${ }^{16}$ E. Aksel, J. S. Forrester, J. L. Jones, P. A. Thomas, K. Page and M. R. Suchomel, Monoclinic crystal structure of polycrystalline $\mathrm{Na}_{0.5} \mathrm{Bi}_{0.5} \mathrm{TiO}_{3}$, Appl. Phys. Lett. 98, 152901 (2011).
${ }^{17}$ Z. Yang, B. Liu, L. Wei and Y. Hou, Structure and electrical properties of $(1-x) \mathrm{Bi}_{0.5} \mathrm{Na}_{0.5} \mathrm{TiO}_{3}-x \mathrm{Bi}_{0.5} \mathrm{~K}_{0.5} \mathrm{TiO}_{3}$ ceramics near morphotropic phase boundary, Mater. Res. Bull. 43, 81 (2008).

${ }^{18}$ J. Kreisel, A. M. Glazer, G. Jones, P. A. Thomas, L. Abello and G. Lucazeau, An X-ray diffraction and Raman spectroscopy investigation of A-site substituted perovskite compounds: The $\left(\mathrm{Na}_{1-x} \mathrm{~K}_{x}\right)_{0.5} \mathrm{Bi}_{0.5} \mathrm{TiO}_{3}(0 \leq x \leq 1)$ solid solution, J. Phys.: Condens. Matter 12, 3267 (2000).

${ }^{19}$ M. Izumi, K. Yamamoto, M. Suzuki, Y. Noguchi and M. Miyayama, Large electric-field-induced strain in $\mathrm{Bi}_{0.5} \mathrm{Na}_{0.5} \mathrm{TiO}_{3^{-}}$ $\mathrm{Bi}_{0.5} \mathrm{~K}_{0.5} \mathrm{TiO}_{3}$ solid solution single crystals, Appl. Phys. Lett. 93, 242903 (2008).

${ }^{20}$ P.-Y. Chen, C.-C. Chou, T.-Y. Tseng and H. H. D. Chen, Comparative study between conventional and microwave sintered leadfree BNKT ceramics, Ferroelectrics 381, 196 (2009).

${ }^{21}$ O. Elkechai, M. Manier and J. P. Mercurio, $\mathrm{Na}_{0.5} \mathrm{Bi}_{0.5} \mathrm{TiO}_{3}$ $\mathrm{K}_{0.5} \mathrm{Bi}_{0.5} \mathrm{TiO}_{3}$ (NBT-KBT) system: A structural and electrical study, Phys. Status Solidi A 157, 499 (1996).

${ }^{22}$ H. Xie, L. Jin, D. Shen, X. Wang and G. Shen, Morphotropic phase boundary, segregation effect and crystal growth in the NBTKBT system, J. Cryst. Growth. 311, 3626 (2009).

${ }^{23}$ B. H. Toby, EXPGUI, a graphical user interface for GSAS, J. Appl. Crystallogr. 34, 210 (2001).

${ }^{24}$ M. Otonicar, S. D. Skapin, M. Spreitzer and D. Suvorov, Compositional range and electrical properties of the morphotropic phase boundary in the $\mathrm{Na}_{0.5} \mathrm{Bi}_{0.5} \mathrm{TiO}_{3}-\mathrm{K}_{0.5} \mathrm{Bi}_{0.5} \mathrm{TiO}_{3}$ system, J. Eur. Ceram. Soc. 30, 971 (2010). 